\title{
Correlation between cloud condensation nuclei concentration and aerosol optical thickness in remote and polluted regions
}

\author{
M. O. Andreae \\ Biogeochemistry Department, Max Planck Institute for Chemistry, Mainz, Germany \\ Received: 7 May 2008 - Published in Atmos. Chem. Phys. Discuss.: 9 June 2008 \\ Revised: 17 September 2008 - Accepted: 10 December 2008 - Published: 26 January 2009
}

\begin{abstract}
A large number of published and unpublished measurements of cloud condensation nuclei $(\mathrm{CCN})$ concentrations and aerosol optical thickness (AOT) measurements have been analyzed. AOT measurements were obtained mostly from the AERONET network, and selected to be collocated as closely as possible to the $\mathrm{CCN}$ investigations. In remote marine regions, $\mathrm{CCN}_{0.4}(\mathrm{CCN}$ at a supersaturation of $0.4 \%$ ) are around $110 \mathrm{~cm}^{-3}$ and the mean $\mathrm{AOT}_{500}$ (AOT at $500 \mathrm{~nm}$ ) is 0.057 . Over remote continental areas, $\mathrm{CCN}$ are almost twice as abundant, while the mean $\mathrm{AOT}_{500}$ is ca. 0.075 . (Sites dominated by desert dust plumes were excluded from this analysis.) Some, or maybe even most of this difference must be because even remote continental sites are in closer proximity to pollution sources than remote marine sites. This suggests that the difference between marine and continental levels must have been smaller before the advent of anthropogenic pollution.

Over polluted marine and continental regions, the $\mathrm{CCN}$ concentrations are about one order of magnitude higher than over their remote counterparts, while AOT is about five times higher over polluted than over clean regions. The average $\mathrm{CCN}$ concentrations from all studies show a remarkable correlation to the corresponding AOT values, which can be expressed as a power law. This can be very useful for the parameterization of CCN concentrations in modeling studies, as it provides an easily measured proxy for this variable, which is difficult to measure directly. It also implies that, at least at large scales, the radiative and microphysical effects of aerosols on cloud physics are correlated and not free to vary fully independently. While the observed strong empirical correlation is remarkable, it must still be noted that there
\end{abstract}

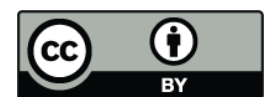

Correspondence to: M. O. Andreae (andreae@mpch-mainz.mpg.de) is about a factor-of-four range of $\mathrm{CCN}$ concentrations at a given AOT, and that there remains considerable room for improvement in remote sensing techniques for $\mathrm{CCN}$ abundance.

\section{Introduction}

The concentration of cloud-active particles, especially in the lower troposphere, has a profound influence on the microphysical processes in clouds, and consequently on many aspects of weather and climate. These interactions have been summarized in a number of recent reviews, addressing in particular the effects of aerosols on climate (Penner et al., 2001; Lohmann and Feichter, 2005; IPCC, 2007) and on cloud processes and precipitation (McFiggans et al., 2006; Rosenfeld, 2006a; IAPSAG, 2007; Andreae and Rosenfeld, 2008). In addition to their cloud microphysical effects, aerosols also modulate cloud formation and convective behavior through their radiative effects, for which aerosol optical thickness (AOT) is a commonly used metric. AOT has the advantage of being readily observed by remote sensing, and AOT measurements are now done routinely from space by several sensors (Kaufman et al., 2002; Yu et al., 2003; Kahn et al., 2007; Kokhanovsky et al., 2007) as well as by ground-based sunphotometer networks (Holben et al., 2001; Kim et al., 2008).

In order to incorporate the effects of cloud condensation nuclei $(\mathrm{CCN})$ in meteorological models at all scales, from large eddy simulation (LES) to global climate models (GCM), knowledge of the spatial and temporal distribution of $\mathrm{CCN}$ in the atmosphere is essential. This information is, however, difficult to obtain from observations. In-situ measurements of $\mathrm{CCN}$ concentrations only provide very localized and sparse information, while the detection of $\mathrm{CCN}$ by remote sensing has not yet been accomplished. This is due

Published by Copernicus Publications on behalf of the European Geosciences Union. 
to the difference in size ranges important for $\mathrm{CCN}$ concentrations on one hand, and for light extinction on the other.

The ability of a particle to nucleate a cloud droplet depends on its size and composition. The latter is now frequently represented by the hygroscopicity factor, $\kappa$, which typically falls in the range of 0.1-0.9 for ambient aerosols (Petters and Kreidenweis, 2007; Andreae and Rosenfeld, 2008). At these values of $\kappa$, particles must have diameters larger than about $40-70 \mathrm{~nm}$ in order to activate at the highest supersaturations commonly found in clouds (up to about $0.6 \%$ ). This therefore represents the lower boundary of the size range containing $\mathrm{CCN}$-active particles. The upper boundary is effectively defined by the sharp decrease in the number concentration of particles as a function of size, so that usually only a minor fraction of CCN is in the size range above some 200$300 \mathrm{~nm}$ diameter (Seinfeld and Pandis, 1998). The maximum of the CCN size distribution thus typically falls in the range of about $70-200 \mathrm{~nm}$. This range is well below the maximum in the Mie scattering efficiency function for light with a wavelength of about $500 \mathrm{~nm}$, which is most commonly used to represent the aerosol optical thickness (AOT) of the atmospheric column. In contrast, the maximum of the scattering and extinction efficiency functions often falls near or above the maximum of the mass size distribution of the aerosol in the range between 400 and $1000 \mathrm{~nm}$, so that this part of the size distribution usually has the strongest influence on the $\mathrm{AOT}_{500}$ values. In regions with high loadings of dust and seasalt aerosol, the coarse mode ( $>1 \mu \mathrm{m}$ diameter) may also contribute strongly to $\mathrm{AOT}_{500}$ (Mulcahy et al., 2008; Remer et al., 2008).

The disconnect between the parts of the aerosol size spectrum dominating the $\mathrm{CCN}$ abundance and those dominating visible light extinction suggests that correlations between these two variables may not be very strong. Consequently, the use of remote sensing measurements for the estimation of CCN abundances has been considered difficult (Gasso and Hegg, 2003; Ghan et al., 2006; Kapustin et al., 2006; Rosenfeld, 2006b). Further complications arise from the fact that column-integrated properties, such as AOT, are not necessarily representative of surface or near-surface properties such as the concentration of aerosol particles and $\mathrm{CCN}$ in the boundary layer. Variability in the scale height of the vertical aerosol distribution and the existence of aerosol layers aloft can introduce substantial variability in the relationship between column and surface properties. Changes in RH can result in pronounced variations in AOT even while dry aerosol concentrations and $\mathrm{CCN}$ concentrations remain the same, adding further variability into a potential relationship between AOT and boundary-layer CCN concentrations.

The motivation for the present study came from a discussion about the choice of variables that would be appropriate as drivers in global models of aerosol effects on clouds and climate, and the ranges of values that would need to be considered for these variables. We selected AOT as a proxy for the radiative effect of aerosols on cloud forma- tion and the production of convectively available potential energy (CAPE) at the surface. $\mathrm{CCN}_{0.4}$ was chosen to represent microphysical effects. As no global compilation of the available data for these properties was available, and because a substantial amount of new data for both variables has recently become available, I decided to make an empirical investigation of their correlation in collocated (or at least nearly collocated) data sets. A conceptual analysis of the combined radiative and microphysical effects of aerosols on clouds, based in part on the results reported here, has recently been published (Rosenfeld et al., 2008).

\section{Methods}

Most AOT values were obtained from the AERONET database publicly available on the Internet at http://aeronet. gsfc.nasa.gov/. These data have the advantage of being available from a global network of stations with consistent processing algorithms and quality control (Dubovik et al., 2000). In most cases, I was able to use Level 2.0 data, which are fully calibrated and cloud-screened. In some instances, only Level 1.5 data were available, which have been cloudscreened, but do not yet have the final calibration applied. These cases, and the few cases where other sunphotometer data were used, have been indicated in Tables 1 and 2. I calculated the statistics reported in Tables 1 and 2 based on the daily average data reported in the AERONET database, in order to avoid introducing bias from variability in the number of measurements available on individual days. I chose not to use MODIS AOT data for this analysis because of persisting uncertainties regarding the absolute accuracy of this data $(\mathrm{Li}$ et al., 2007).

I chose to use AOT values at $500 \mathrm{~nm}\left(\mathrm{AOT}_{500}\right)$, because they are most commonly available and most frequently used as a metric for aerosol burdens. When only measurements at other wavelengths were available, I chose the nearest available wavelength and made an adjustment using the appropriate Ångstrom exponent.

To represent CCN concentrations, I used the set of particles that activate at a supersaturation of $0.4 \%$, a value commonly used for convective clouds. Many of the data were taken from the literature, and have been obtained using a variety of instruments about which details can be found in the original papers referenced in the Tables. Our own previously unpublished measurements were obtained with the static chamber counter described by Frank et al. (2007) and the DMT continuous-flow counter using the techniques discussed in Rose et al. (2008). When only data at supersaturations other than $0.4 \%$ were available, they were adjusted to $0.4 \%$ using the conventional power law formulation for the dependence of $\mathrm{CCN}$ concentrations on supersaturation $\left(\left[\mathrm{CCN}_{S}\right]=\left[\mathrm{CCN}_{1.0}\right]^{*} \mathrm{~S}^{k}\right)$. The exponent, $k$, was either derived from the data, or where this was not possible, I used default values of 0.5 for continental and 0.4 for marine sites. 
Table 1. $\mathrm{CCN}_{0.4}, \mathrm{CN}$, and $\mathrm{AOT}_{500}$ measurements from the Amazon Basin.

\begin{tabular}{|c|c|c|c|c|c|}
\hline Campaign, Time, Season & $\mathrm{CCN}_{0.4} \mathrm{~cm}^{-3}$ & $\mathrm{CN} \mathrm{cm}^{-3}$ & AOT $(500 \mathrm{~nm})$ & Location & Reference \\
\hline \multicolumn{6}{|l|}{ Clean conditions } \\
\hline $\begin{array}{l}\text { CLAIRE-1998 } \\
\text { Feb-Mar 1998, wet }\end{array}$ & $190 \pm 130$ & $460 \pm 320$ & $0.089 \pm 0.020^{*}$ & $\begin{array}{l}\text { Balbina, Amazonas } \\
01.92 \mathrm{~S}, 059.49 \mathrm{~W}\end{array}$ & $\begin{array}{l}\text { Roberts et al. (2001); } \\
\text { Mar-Jun 2000-2001 }\end{array}$ \\
\hline $\begin{array}{l}\text { LBA-EUSTACH } \\
\text { Apr 1999, wet }\end{array}$ & $155 \pm 65^{\mathrm{a}}$ & $380 \pm 160$ & 0.048 & $\begin{array}{l}\text { Rebio Jarú, Rondonia } \\
10.08 \mathrm{~S}, 061.93 \mathrm{~W}\end{array}$ & Guyon et al. (2003) \\
\hline $\begin{array}{l}\text { LBA-EUSTACH } \\
\text { Jan-Mar, Nov 1999, wet }\end{array}$ & $230 \pm 100^{\mathrm{a}}$ & - & $0.121 \pm 0.033^{*}$ & $\begin{array}{l}\text { Ji Paraná, Rondonia } \\
10.88 \mathrm{~S}, 061.94 \mathrm{~W}\end{array}$ & Williams et al. (2002) \\
\hline $\begin{array}{l}\text { CLAIRE-2001 } \\
\text { July 2001, late wet }\end{array}$ & $190 \pm 90$ & $530 \pm 430$ & $0.082 \pm 0.008^{*}$ & $\begin{array}{l}\text { Balbina, Amazonas } \\
01.92 \mathrm{~S}, 059.49 \mathrm{~W}\end{array}$ & Rissler et al. (2004) \\
\hline $\begin{array}{l}\text { SMOCC } \\
\text { Oct } 2002 \text {, late dry }\end{array}$ & $205 \pm 40$ & $500 \pm 100$ & - & $\begin{array}{l}\text { NW of Cruzeiro do Sul, AM } \\
\sim 07 \mathrm{~S}, 073 \mathrm{~W}\end{array}$ & Andreae et al. (2004) \\
\hline $\begin{array}{l}\text { AMAZE-2008 } \\
\text { Feb-Mar 2008, wet }\end{array}$ & $138 \pm 94^{\mathrm{a}}$ & $336 \pm 228$ & - & $\begin{array}{l}\mathrm{N} \text { of Manaus } \\
02.60 \mathrm{~S}, 060.21 \mathrm{~W}\end{array}$ & S. Gunthe, J. Schneider, unpubl. \\
\hline $\begin{array}{l}\text { AERONET } \\
1993-2006\end{array}$ & - & - & $0.093 \pm 0.06$ & Amazon Basin forest & Schafer et al. (2008) \\
\hline \multicolumn{6}{|l|}{ Smoky conditions } \\
\hline $\begin{array}{l}\text { LBA-EUSTACH Sep-Oct 1999, dry } \\
\text { LBA-EUSTACH Sep-Oct 1999, dry } \\
\text { LBA-EUSTACH Oct 1999, late dry } \\
\text { CLAIRE-2001 Jul 2001, late wet } \\
\text { SMOCC Sep-Oct 2002, dry } \\
\text { AERONET 1993-2006 }\end{array}$ & $\begin{array}{c}1000-4000^{\mathrm{a}} \\
1300-7500^{\mathrm{a}} \\
650-2000^{\mathrm{a}} \\
400-1000 \\
1000-4000 \\
-\end{array}$ & $\begin{array}{c}2000-8000 \\
2500-15000 \\
- \\
800-2000 \\
2000-8000 \\
-\end{array}$ & $\begin{array}{c}0.80 \pm 0.24 \\
0.91 \\
0.90^{*} \\
0.089 \pm 0.023^{*} \\
0.95^{*} \\
0.90 \pm 0.63\end{array}$ & $\begin{array}{l}\text { Rebio Jarú, Rondonia } 10.08 \mathrm{~S}, 061.93 \mathrm{~W} \\
\text { Fazenda Nossa Senhora, RO } 10.76 \mathrm{~S}, 062.36 \mathrm{~W} \\
\text { Ji Paraná, Rondonia } 10.88 \mathrm{~S}, 061.94 \mathrm{~W} \\
\text { Balbina, Amazonas } 01.92 \mathrm{~S}, 059.49 \mathrm{~W} \\
\text { Rondonia and Mato Grosso } 99-13 \mathrm{~S}, 056-064 \mathrm{~W} \\
\text { Southern Amazon Basin forest }\end{array}$ & $\begin{array}{l}\text { Guyon et al. (2003) } \\
\text { Artaxo et al. (2002) } \\
\text { Williams et al. (2002) } \\
\text { Rissler et al. (2004) } \\
\text { Andreae et al. (2004) } \\
\text { Schafer et al. (2008) }\end{array}$ \\
\hline \multicolumn{6}{|l|}{ Smoke plumes } \\
\hline SMOCC Oct 2002, dry & $10000-22000$ & $20000-44000$ & - & Rondonia and Mato Grosso 98-13 S, 056-064 W & Andreae et al. (2004) \\
\hline
\end{tabular}

* Data from the AERONET website. When the AERONET data period differs from the campaign period, it is indicated in the Reference column. Other AOT data are from on-site measurements.

${ }^{\text {a }} \mathrm{CCN}_{0.4}$ calculated from $\mathrm{CN}$ using $\mathrm{CCN}_{0.4} / \mathrm{CN}$ ratio (see text).

In order to increase the available database, I deduced $\mathrm{CCN}$ concentrations from measurements of aerosol size spectra for some cases where direct CCN measurements were not available. For this purpose, I used the lower cutoff diameters specified in the Comment column in Table 2, chosen based either on measurements of these cutoff diameters from the same site or from similar locations, or obtained using appropriate values of $\kappa$, and the relationships between $\kappa$ and the activation diameter given by Petters and Kreidenweis (2007). Obviously, this introduces additional uncertainty into our estimates, which is in each case a function of the accuracy of the estimate of the cutoff diameter and the shape of the size distribution. In unfavorable cases, this uncertainty may be large (up to about 50\%). In most cases, however, the maximum of the size distributions at our rural and remote stations was well above the range of possible cutoff diameters (50-90 nm for the range of $\kappa$ and supersaturations considered here), and therefore the resulting uncertainty is likely not greater than what one must accept when using literature data from a great variety of groups and instruments. Since we are looking only for fairly broad relationships and consider a wide range of datasets and conditions, it is unlikely that this approach would introduce a systematic bias.
In addition to data sets where corresponding pairs of AOT and $\mathrm{CCN}$ data were available, some values where only one of the variables was measured have been added to the Tables for information purposes. Furthermore, condensation nuclei $(\mathrm{CN})$ concentrations have been provided in the Tables, when available. These data have been obtained by a variety of instruments, with different lower cut-off diameters, and thus some caution must be exercised when comparing their values.

\section{Results and discussion}

For this study, I have separated the available data into four general regimes, continental-remote, continental-polluted, marine-remote and marine-polluted. I have excluded the analysis of dust-dominated regions downwind of the major dust source regions from our analysis, because there is little or no collocated CCN and AOT data available, and because dominance of the coarse mode in these regions precludes a meaningful relationship between $\mathrm{CCN}$ and AOT.

An emphasis on looking at remote regions came from our interest in estimating the pre-human aerosol loading of the 
Table 2. $\mathrm{CCN}_{0.4}, \mathrm{CN}$, and $\mathrm{AOT}_{500}$ measurements from remote and polluted, marine and continental environments. Data are presented as means and standard deviations ( $\mathrm{mmm} \pm \mathrm{sss}$ ) or medians and quartile ranges [nnn (lll-uuu)]. " $\mathbb{E}:$ : identifies the AERONET site from which the AOT data are taken. Where the CCN and AOT data are from different locations, this is indicated in the Position column.

\begin{tabular}{|c|c|c|c|c|c|c|c|}
\hline Location, Time & $\begin{array}{l}\mathrm{CCN}_{0.4} \\
{\left[\mathrm{~cm}^{-3}\right]}\end{array}$ & $\begin{array}{c}\mathrm{CN} \\
{\left[\mathrm{cm}^{-3}\right]}\end{array}$ & $\mathrm{CCN}_{0.4} / \mathrm{CN}$ & $\mathrm{AOT}_{500}$ & Comment & Position & Reference \\
\hline \multicolumn{8}{|l|}{ Clean conditions, marine } \\
\hline $\begin{array}{l}\text { Cape Grim } \\
\text { summer } \\
\text { winter }\end{array}$ & $\begin{array}{c}119 \pm 32 \\
46 \pm 11\end{array}$ & $\begin{array}{l}570 \pm 80 \\
153 \pm 37\end{array}$ & $\begin{array}{l}0.21 \\
0.30\end{array}$ & $\begin{array}{l}0.048 \pm 0.010 \\
0.015 \pm 0.010\end{array}$ & AOT data from CGO station & $40.68 \mathrm{~S}, 144.69 \mathrm{E}$ & $\begin{array}{l}\text { Gras (1990); J. Gras, unpubl.; } \\
\text { Wilson and Forgan (2002) }\end{array}$ \\
\hline $\begin{array}{l}\text { Southern Ocean, off Tasmania } \\
\text { summer } \\
\text { winter }\end{array}$ & $\begin{array}{l}90 \pm 20 \\
27 \pm 10\end{array}$ & $\begin{array}{l}266 \pm 150 \\
210 \pm 190\end{array}$ & $\begin{array}{l}0.34 \\
0.13\end{array}$ & $\begin{array}{l}0.048 \pm 0.010 \\
0.015 \pm 0.010\end{array}$ & $\begin{array}{l}\text { aircraft, baseline } \\
\text { AOT data from CGO station }\end{array}$ & $\begin{array}{l}\sim 41-42 \mathrm{~S}, 144 \mathrm{E} \\
40.68 \mathrm{~S}, 144.69 \mathrm{E}\end{array}$ & Yum and Hudson (2004) \\
\hline $\begin{array}{l}\text { S. Indian Ocean } \\
\text { Mar-Apr } 2001\end{array}$ & $210 \pm 140$ & - & - & $0.065(0.048,0.091)^{\mathrm{b}}$ & $\begin{array}{l}\text { off S. Africa } \\
\text { Æ: Reunion }\end{array}$ & $20.88 \mathrm{~S}, 055.48 \mathrm{E}$ & Ross et al. (2003) \\
\hline $\begin{array}{l}\text { S. Trop. Indian Oc. } \\
\text { Feb/Mar } 1999\end{array}$ & $150 \pm 20$ & $361 \pm 31$ & 0.42 & $0.058 \pm 0.035^{\mathrm{b}}$ & $\begin{array}{l}\text { INDOEX } \\
\text { Æ: Reunion }\end{array}$ & $\begin{array}{l}\sim 3-8 \mathrm{~S}, \sim 072-074 \mathrm{E} \\
20.88 \mathrm{~S}, 055.48 \mathrm{E}\end{array}$ & Hudson and Yum (2002) \\
\hline $\begin{array}{l}\text { Trop. S. Pacific } \\
\text { Nov/Dec 2003/04 }\end{array}$ & $240 \pm 90$ & $350 \pm 150$ & 0.69 & $0.078(0.060,0.010)^{\mathrm{b}}$ & $\begin{array}{l}\text { STRATUS 2003/04 } \\
\text { Æ: Tahiti }\end{array}$ & $\begin{array}{l}\sim 11-28 \mathrm{~S}, 71-90 \mathrm{~W} \\
17.58 \mathrm{~S}, 149.60 \mathrm{~W}\end{array}$ & Tomlinson et al. (2007) \\
\hline $\begin{array}{l}\text { Trop. N. Pacific } \\
\text { Jul-Aug } 1990\end{array}$ & $80 \pm 50$ & $180 \pm 110$ & 0.44 & $0.065 \pm 0.026$ & $\begin{array}{l}\text { near Hawaii } \\
\text { Æ: Lanai, Jul/Aug 1997-2003 }\end{array}$ & $20.73 \mathrm{~N}, 156.92 \mathrm{~W}$ & Hudson (1993) \\
\hline $\begin{array}{l}\text { Temp. S. Pacific } \\
\text { Nov-Dec } 1995\end{array}$ & $108 \pm 44$ & $330 \pm 70$ & 0.33 & - & ACE1 & $\sim 41-51 \mathrm{~S}, 138-150 \mathrm{E}$ & Hudson et al. (1998) \\
\hline $\begin{array}{l}\text { Temp. N. Pacific } \\
\text { winter } 1988-1990 \\
\text { summer } 1989\end{array}$ & $\begin{array}{l}23 \\
78\end{array}$ & $\begin{array}{l}252 \\
594\end{array}$ & $\begin{array}{l}0.09 \\
0.13\end{array}$ & - & off Washington & $\sim 47 \mathrm{~N}, 128 \mathrm{~W}$ & Hegg et al. (1991) \\
\hline $\begin{array}{l}\text { Temp. N. Pacific } \\
\text { Jun-Jul } 1987\end{array}$ & $55 \pm 35$ & $170 \pm 80$ & 0.32 & $0.072(0.058,0.096)$ & $\begin{array}{l}\text { FIRE, below stratus } \\
\text { Æ: San Nicolas Isl. }\end{array}$ & $\begin{array}{l}\sim 31 \mathrm{~N}, 122 \mathrm{~W} \\
32.26 \mathrm{~N}, 119.49 \mathrm{~W}\end{array}$ & Hudson and Frisbie (1991a) \\
\hline $\begin{array}{l}\text { Arctic Ocean } \\
\text { May } 1998\end{array}$ & $\begin{array}{c}54 \pm 21 \\
180 \pm 30\end{array}$ & $\begin{array}{c}161 \pm 125 \\
395 \pm 95\end{array}$ & $\begin{array}{l}0.34 \\
0.46\end{array}$ & $\begin{array}{l}- \\
-\end{array}$ & $\begin{array}{l}\text { below low cloud } \\
\text { no low cloud }\end{array}$ & $\sim 76 \mathrm{~N}, 165 \mathrm{~W}$ & Yum and Hudson (2001) \\
\hline $\begin{array}{l}\text { Temp. North Atlantic } \\
\text { Mace Head }\end{array}$ & $120 \pm 50$ & - & - & - & little seasonality & $53.33 \mathrm{~N}, 009.90 \mathrm{~W}$ & Jennings et al. (1998) \\
\hline $\begin{array}{l}\text { N. Atl., Mace Head } \\
\text { summer } \\
\text { winter }\end{array}$ & $\begin{array}{l}81 \pm 11 \\
96 \pm 4 \\
69 \pm 6\end{array}$ & - & - & - & clean marine periods & $53.33 \mathrm{~N}, 009.90 \mathrm{~W}$ & Reade et al. (2006) \\
\hline $\begin{array}{l}\text { Temp. N. Atlantic } \\
\text { June } 1992\end{array}$ & $155 \pm 50$ & $380 \pm 150$ & 0.41 & - & ASTEX & $\sim 30-35 \mathrm{~N}, 18-25 \mathrm{~W}$ & Hudson and Xie (1999) \\
\hline $\begin{array}{l}\text { Temp. N. Atlantic } \\
4 \text { Jul } 1997\end{array}$ & $190 \pm 50$ & $910 \pm 160$ & 0.21 & $0.095 \pm 0.018$ & $\begin{array}{l}\text { ACE-2 } \\
\text { Æ: Tenerife }\end{array}$ & $\begin{array}{l}\sim 32-38 \mathrm{~N}, 011-013 \mathrm{~W} \\
28.03 \mathrm{~N} 016.63 \mathrm{~W}\end{array}$ & Johnson et al. (2000) \\
\hline $\begin{array}{l}\text { Puerto Rico } \\
\text { 13-14 Dec } 2004 \\
1-9 \text { Jan } 2005\end{array}$ & $\begin{array}{l}108 \pm 54 \\
101 \pm 42\end{array}$ & $290 \pm 100$ & 0.37 & $\begin{array}{l}0.065 \pm 0.020 \\
0.060 \pm 0.016\end{array}$ & $\begin{array}{l}\text { Æ: Cape San Juan } \\
\text { Dec } 2005 \\
\text { 1-9 Jan } 2007\end{array}$ & $18.38 \mathrm{~N}, 065.62 \mathrm{~W}$ & $\begin{array}{l}\text { Allan et al. (2008) } \\
\text { G. Frank, unpubl. }\end{array}$ \\
\hline $\begin{array}{l}\text { Caribbean Sea } \\
\text { Aug } 1965\end{array}$ & 93 & - & - & - & aircraft, $\mathrm{BL}$ ave. & $\sim 17 \mathrm{~N}, 066 \mathrm{~W}$ & Squires and Twomey (1966) \\
\hline $\begin{array}{l}\text { Tropical Atlantic } \\
\text { Oct/Nov } 1990\end{array}$ & $90 \pm 40$ & $320 \pm 120$ & 0.28 & - & Atlantic transect & $\sim 10 \mathrm{~N}-30 \mathrm{~S}, 020-040 \mathrm{~W}$ & Schäfer et al. (1993) \\
\hline Average & $107 \pm 56$ & $350 \pm 200$ & $0.32 \pm 0.15$ & $0.057 \pm 0.023$ & & & \\
\hline
\end{tabular}

${ }^{\mathrm{a}}$ median

b AERONET level 1.5 data 
Table 2. Continued.

\begin{tabular}{|c|c|c|c|c|c|c|c|}
\hline Location, Time & $\begin{array}{l}\mathrm{CCN}_{0.4} \\
{\left[\mathrm{~cm}^{-3}\right]}\end{array}$ & $\begin{array}{c}\mathrm{CN} \\
{\left[\mathrm{cm}^{-3}\right]}\end{array}$ & $\mathrm{CCN}_{0.4} / \mathrm{CN}$ & $\mathrm{AOT}_{500}$ & Comment & Position & Reference \\
\hline \multicolumn{8}{|c|}{ Clean conditions, continental } \\
\hline Amazon Basin & $185 \pm 120$ & $440 \pm 100$ & 0.41 & $0.082 \pm 0.020$ & clean conditions & & Average from Table 1 \\
\hline \multicolumn{8}{|l|}{ Laramie, Wyoming } \\
\hline Summer & $280 \pm 100$ & $6800 \pm 3800$ & 0.04 & $0.117(0.081-0.179)$ & & $\sim 41 \mathrm{~N}, 104 \mathrm{~W}(\mathrm{CCN})$ & Delene and Deshler (2001) \\
\hline Winter & $92 \pm 12$ & $3180 \pm 1120$ & 0.03 & $0.069(0.051-0.106)$ & Æ: Missoula, MT & $46.9 \mathrm{~N}, 114.08 \mathrm{~W}(Æ)$ & \\
\hline $\begin{array}{l}\text { Yukon Valley } \\
\text { Feb } 1972\end{array}$ & $90 \pm 10$ & - & - & - & - & $\sim 66 \mathrm{~N}, 148 \mathrm{~W}$ & Hoppel et al. (1973) \\
\hline $\begin{array}{l}\text { Colorado Plains } \\
\text { Summer } 1965\end{array}$ & 280 & - & - & - & somewhat polluted & $\sim 40 \mathrm{~N}, 105 \mathrm{~W}$ & Squires and Twomey (1966) \\
\hline $\begin{array}{l}\text { Fort McMurray } \\
\text { Canada }\end{array}$ & - & - & - & $0.057(0.034-0.089)$ & Æ: Fort McMurray & $56.75 \mathrm{~N}, 111.48 \mathrm{~W}$ & \\
\hline $\begin{array}{l}\text { Pallas, Finland } \\
\text { Apr 2000-Feb } 2002\end{array}$ & $152 \pm 33$ & 410 & 0.37 & - & $\mathrm{CN}>80 \mathrm{~nm}$, activated particles & & Komppula et al. (2005) \\
\hline $\begin{array}{l}\text { Pallas, Finland } \\
\text { winter } \\
\text { summer }\end{array}$ & 235 & $\begin{array}{c} \\
\quad 810 \\
\sim 200 \\
\sim 1200\end{array}$ & 0.29 & - & $\mathrm{CN}>80 \mathrm{~nm}$ & $67.97 \mathrm{~N}, 024.12 \mathrm{E}$ & Lihavainen et al. (2003) \\
\hline $\begin{array}{l}\text { Hyytiälä, Finland } \\
\text { 1996-2007 }\end{array}$ & $354^{\mathrm{a}}$ & 2125 & 0.17 & - & median, $\mathrm{CN}>65 \mathrm{~nm}$ & $61.85 \mathrm{~N}, 024.30 \mathrm{E}$ & M. Kulmala, unpubl. \\
\hline $\begin{array}{l}\text { Siberia, ZOTTO } \\
\text { Sep 2006-Sep } 2007\end{array}$ & $\begin{array}{c}187 \\
(109,297)\end{array}$ & $283(173,446)$ & 0.66 & $0.081(0.053-0.120)$ & $\begin{array}{l}\mathrm{CN}>60 \mathrm{~nm}, \sigma_{a}<2 \mathrm{Mm}^{-1} \\
\text { Æ: Yakutsk }\end{array}$ & $\begin{array}{l}60.80 \mathrm{~N}, 089.35 \mathrm{E} \\
61.66 \mathrm{~N}, 129.37 \mathrm{E}(Æ)\end{array}$ & W. Birmili, J. Heintzenberg, unpubl. \\
\hline $\begin{array}{l}\text { South Africa } \\
1 \text { Sep } 2000\end{array}$ & $137 \pm 63$ & - & - & $0.045 \pm 0.013$ & $\begin{array}{l}\text { S. Highveld, dry season } \\
\text { Æ: Bethlehem }\end{array}$ & $28.25 \mathrm{~S}, 028.33 \mathrm{E}(Æ)$ & Ross et al. (2003) \\
\hline Average & $200 \pm 90$ & $2010 \pm 2370$ & $0.38 \pm 0.18$ & $0.075 \pm 0.025$ & & & \\
\hline
\end{tabular}

a median

b AERONET level 1.5 data

atmosphere, which is of relevance to understanding the human perturbation (Andreae, 2007a). At the present time, the most remote, and therefore probably closest to pristine, continental regions are found in Amazonia and in parts of northern North America and Siberia. Because of the seasonal heavy pollution of the Amazonian atmosphere with biomass smoke, this area also provides the opportunity to study very clean and highly polluted conditions within the same region.

\section{Amazonia}

Even in an area as remote as the Amazon Basin, the presentday aerosol population is influenced by anthropogenic emissions, which are mostly from biomass burning. When the emission sources are large, as during the regional fire season, or located nearby, this influence can be readily identi- fied. On the other hand, emissions arriving by long-range transport, especially from Africa, can have a significant influence on the aerosol population over Amazonia, even when there is little fire activity in the Amazon Basin (Prospero et al., 1981; Talbot et al., 1990; Swap et al., 1992; Formenti et al., 2001). For this reason, the $\mathrm{CCN}$ and $\mathrm{CN}$ concentrations summarized in Table 1 must always be considered as upper limits for the pristine values. The first CCN measurements in Amazonia were obtained during CLAIRE-98 by Roberts et al. (2001) at Balbina near Manaus. Subsequently, CCN and $\mathrm{CN}$ measurements were made as part of the LBA-EUSTACH and SMOCC programs (Andreae et al., 2002; Andreae et al., 2004). In the course of a thorough investigation of the CCN counter used in the study of Roberts et al. (2001), it was found that the supersaturations in that study had been overestimated (Frank et al., 2007). Therefore, the values were 
Table 2. Continued.

\begin{tabular}{|c|c|c|c|c|c|c|c|}
\hline Location, Time & $\begin{array}{l}\mathrm{CCN}_{0.4} \\
{\left[\mathrm{~cm}^{-3}\right]}\end{array}$ & $\underset{\left[\mathrm{cm}^{-3}\right]}{\mathrm{CN}}$ & $\mathrm{CCN}_{0.4} / \mathrm{CN}$ & $\mathrm{AOT}_{500}$ & Comment & Position & Reference \\
\hline \multicolumn{8}{|l|}{ Polluted conditions, marine } \\
\hline $\begin{array}{l}\text { North Atlantic } \\
\text { Mace Head }\end{array}$ & $630 \pm 380$ & - & - & - & polluted conditions & $53.33 \mathrm{~N}, 009.90 \mathrm{~W}$ & Jennings et al. (1998) \\
\hline $\begin{array}{l}\text { NW Atlantic, off } \\
\text { Nova Scotia } \\
\text { Aug-Sep } 1993\end{array}$ & $1170 \pm 700$ & $4420 \pm 6160$ & 0.26 & $0.15 \pm 0.06$ & $\begin{array}{l}\text { NARE } \\
\text { Æ: Keijmkuijk } \\
\text { (Aug-Sep 1998-1999) }\end{array}$ & $\begin{array}{l}43.85 \mathrm{~N}, 066.12 \mathrm{~W} \\
44.38 \mathrm{~N}, 065.28 \mathrm{~W}\end{array}$ & Liu et al. (1996) \\
\hline $\begin{array}{l}\text { Temp. N Atlantic } \\
\text { June } 1992\end{array}$ & $840 \pm 340$ & $1390 \pm 550$ & 0.60 & - & ASTEX & $\sim 30-35 \mathrm{~N}, 18-25 \mathrm{~W}$ & Hudson and Xie (1999) \\
\hline $\begin{array}{l}\text { Temp. N Atlantic } \\
\text { 16-18 Jul } 1997 \\
\text { 23-24 Jul } 1997\end{array}$ & $\begin{array}{l}660 \pm 200 \\
830 \pm 130\end{array}$ & $\begin{array}{l}2270 \pm 800 \\
1710 \pm 200\end{array}$ & $\begin{array}{l}0.29 \\
0.49\end{array}$ & $0.32 \pm 0.09$ & ACE-2 & $\begin{array}{l}\sim 33-40 \mathrm{~N}, 011-014 \mathrm{~W} \\
\sim 30-38 \mathrm{~N}, 011-013 \mathrm{~W}\end{array}$ & $\begin{array}{l}\text { Osborne et al. (2000) } \\
\text { Wood et al. (2000) }\end{array}$ \\
\hline $\begin{array}{l}\text { Temp. NW Pacific } \\
\text { Gosan, Korea }\end{array}$ & $1570 \pm 500$ & $3510 \pm 1790$ & 0.45 & $0.28+0.02$ & ABC-EAREX & $33.29 \mathrm{~N}, 126.16 \mathrm{~W}$ & Yum et al. (2007) \\
\hline $\begin{array}{l}\text { Temp. NW Pacific } \\
\text { Anmyeon Isl., KR } \\
1-22 \text { May } 2004\end{array}$ & $1670 \pm 390$ & $3980 \pm 970$ & 0.42 & $0.38 \pm 0.17$ & Æ: Amnyon & $36.54 \mathrm{~N}, 126.33 \mathrm{E}$ & Yum et al. (2005) \\
\hline $\begin{array}{l}\text { Indian Ocean, NH } \\
\text { Feb/Mar } 1999\end{array}$ & $1100 \pm 100$ & $1810 \pm 40$ & 0.61 & $0.39 \pm 0.17$ & $\begin{array}{l}\text { INDOEX } \\
\text { Æ: Kaashidoo }\end{array}$ & $\begin{array}{l}\sim 0-4 \mathrm{~N}, \sim 072-074 \mathrm{E} \\
4.96 \mathrm{~N}, 073.47 \mathrm{E}\end{array}$ & Hudson and Yum (2002) \\
\hline Average & $1060 \pm 400$ & $2700 \pm 1200$ & $0.44 \pm 0.14$ & $0.30 \pm 0.10$ & & & \\
\hline \multicolumn{8}{|l|}{ Polluted conditions, continental } \\
\hline Mace Head, Ireland & $370 \pm 70$ & - & - & - & polluted continental & & Reade et al. (2006) \\
\hline $\begin{array}{l}\text { Amazon, } \\
\text { smoky season }\end{array}$ & $2500 \pm 1500$ & $5000 \pm 3000$ & 0.50 & $0.90 \pm 0.30$ & southern part of Amazon Basin & $6-17 \mathrm{~S}, 45-70 \mathrm{~W}$ & Average from Table 1 \\
\hline Feldberg, near Frankfurt, Germany & $\begin{array}{c}2300 \pm 1000 \\
1400 \pm 800\end{array}$ & $\begin{array}{l}4650 \pm 1800 \\
5700 \pm 4700\end{array}$ & $\begin{array}{l}0.49 \\
0.25\end{array}$ & $\begin{array}{l}0.30 \pm 0.16 \\
0.20 \pm 0.13\end{array}$ & $\begin{array}{l}20 \text { Jul-11 Aug } 2004 \\
22 \text { Jun-6 Jul } 2005 \\
\text { Æ: Mainz, summer 2004/06 }\end{array}$ & $\begin{array}{l}\text { freq. nucleation events } \\
50.00 \mathrm{~N}, 008.30 \mathrm{E}\end{array}$ & U. Dusek, unpubl. \\
\hline $\begin{array}{l}\text { Hohenpeissenberg } \\
\text { Germany } \\
\text { 1998-2000 }\end{array}$ & $1120 \pm 670$ & $3130 \pm 2580$ & 0.36 & $0.10 \pm 0.07$ & $\begin{array}{l}\mathrm{CCN}_{0.4}=\mathrm{CN}(>60 \mathrm{~nm}) \\
1999-2001, \mathrm{GAW}\end{array}$ & $47.80 \mathrm{~N}, 011.12 \mathrm{E}$ & $\begin{array}{l}\text { A. Wiedensohler, unpubl. } \\
\text { Birmili et al. (2003) } \\
\text { GAW Brief Nr. } 9\end{array}$ \\
\hline $\begin{array}{l}\text { South Africa } \\
\text { 1999-2001 }\end{array}$ & $740 \pm 460$ & - & - & $0.17 \pm 0.15$ & wet and dry seasons & $\sim-18-30 \mathrm{~S}, 25-32 \mathrm{E}$ & Ross et al. (2003) \\
\hline $\begin{array}{l}\text { Reno, Nevada } \\
\text { Dec 1988-May } 1990\end{array}$ & $1310 \pm 580$ & $8790 \pm 2000$ & 0.15 & - & & & Hudson and Frisbie (1991b) \\
\hline $\begin{array}{l}\text { New Hampshire } \\
\text { Aug } 2004\end{array}$ & $1090 \pm 350$ & $\sim 5000$ & 0.22 & $0.24 \pm 0.21$ & $\begin{array}{l}\text { rural site } \\
Æ: \text { Billerica }\end{array}$ & $42.53 \mathrm{~N}, 71.27 \mathrm{~W}$ & Medina et al. (2007) \\
\hline $\begin{array}{l}\text { North Carolina } \\
\text { Duke Forest, Jul } 2003\end{array}$ & 930 & 3400 & 0.27 & $0.38 \pm 0.16$ & Æ: Walker Branch & $35.96 \mathrm{~N}, 84.29 \mathrm{~W}$ & Stroud et al. (2007) \\
\hline $\begin{array}{l}\text { Gosan, Korea } \\
11 \text { Mar-9 Apr } 2005\end{array}$ & $2010 \pm 950$ & $5600 \pm 3500$ & 0.36 & $0.35 \pm 0.31$ & ABC-EAREX & $33.29 \mathrm{~N}, 126.16 \mathrm{~W}$ & Yum et al. (2007) \\
\hline $\begin{array}{l}\text { Anmyeon Isl., KR } \\
\text { 1-22 May } 2004\end{array}$ & $3350 \pm 980$ & $8310 \pm 1780$ & 0.40 & $0.50 \pm 0.24$ & Æ: Amnyon & $36.54 \mathrm{~N}, 126.33 \mathrm{E}$ & Yum et al. (2005) \\
\hline $\begin{array}{l}\text { Beijing, } \\
10 \text { Aug-9 Sep } 2006\end{array}$ & $7200 \pm 3000$ & $16200 \pm 8500$ & 0.44 & $0.77 \pm 0.55$ & $\begin{array}{l}\text { Yufa site } \\
\text { Æ: Yufa }\end{array}$ & $39.52 \mathrm{~N}, 116.33 \mathrm{E}$ & D. Rose, unpubl. \\
\hline $\begin{array}{l}\text { Guangzhou Region } \\
\text { NE monsoon } \\
\text { Sep-Oct } 2004\end{array}$ & $7300 \pm 3300$ & $16500 \pm 8800$ & 0.44 & $0.80 \pm 0.38$ & $\begin{array}{l}\mathrm{CN}>60 \mathrm{~nm} \\
\text { Xinken site } \\
Æ: \text { Hong Kong Poly }\end{array}$ & $\begin{array}{l}22.60 \mathrm{~N}, 113.60 \mathrm{E} \\
22.30 \mathrm{~N}, 114.18 \mathrm{E}\end{array}$ & A. Wiedensohler, unpubl. \\
\hline $\begin{array}{l}\text { Guangdong rural, } \\
\text { SE monsoon, Jul } 2006\end{array}$ & $9100 \pm 4800$ & $18700 \pm 8200$ & 0.49 & $0.68 \pm 0.44$ & Æ: Backgarden site & $23.49 \mathrm{~N}, 113.04 \mathrm{E}$ & D. Rose, unpubl. \\
\hline Average & $2900 \pm 2800$ & $8400 \pm 5500$ & $0.36 \pm 0.12$ & $0.45 \pm 0.27$ & & & \\
\hline
\end{tabular}

${ }^{\mathrm{a}}$ median

b AERONET level 1.5 data

Atmos. Chem. Phys., 9, 543-556, 2009

www.atmos-chem-phys.net/9/543/2009/ 
corrected to a critical activation diameter of $85 \mathrm{~nm}$ at a supersaturation of $0.4 \%$, corresponding to a $\mathrm{CCN}_{0.4} / \mathrm{CN}$ ratio for the CLAIRE-98 data of 0.41. This critical diameter appears to be very robust for the clean Amazonian aerosol, and has been found independently in several studies (Rissler et al., 2004; D. Rose, personal communication, 2008). It has therefore also been used, where necessary, for the calculation of $\mathrm{CCN}_{0.4}$ values from $\mathrm{CN}$ values in Table 1 .

The results show surprisingly little difference between wet and dry season measurements in clean conditions. The CLAIRE-98, EUSTACH and AMAZE-08 measurements during the full wet season $(190 \pm 130,155 \pm 65$ and $138 \pm 94 \mathrm{~cm}^{-3}$, respectively) are only moderately lower than the SMOCC-2002 values collected during the full dry season over the western Amazon $\left(205 \pm 40 \mathrm{~cm}^{-3}\right)$. One can therefore conclude that the natural $\mathrm{CCN}_{0.4}$ concentrations over Amazonia are centered around a value no greater than $180 \mathrm{~cm}^{-3}$, with a range of about $80-250 \mathrm{~cm}^{-3}$, and with only a modest seasonal range of about $40 \mathrm{~cm}^{-3}$. Again, I emphasize that even these low values contain some anthropogenic contamination from long-range transport, as shown by the presence of soot particles and a slightly elevated sulfate content that could be related to trans-Atlantic transport (Andreae et al., 1990 and unpublished aerosol composition and SEM data from the campaigns listed above).

During the burning season, pyrogenic aerosols from tens of thousands of fires overwhelm the natural aerosol population. It is not very meaningful to give average concentrations for this situation, as the actual concentration at any given time and place is dictated by the proximity to the fires and the meteorological conditions, and therefore varies over orders of magnitude from near-pristine values to those inside fresh plumes, which can be in the hundreds of thousands per $\mathrm{cm}^{-3}$. Table 1 lists the ranges of typical concentrations observed during several campaigns in the smoky season. One finds that at sites remote from the fires, such as Balbina in northern Amazonas State, $\mathrm{CCN}_{0.4}$ concentrations in the dry season typically reach up to ca. $1000 \mathrm{~cm}^{-3}$, while in the heavily impacted states of Mato Grosso and Rondonia, typical concentrations are in the range of $1000-4000 \mathrm{~cm}^{-3}$.

Aerosol optical thickness measurements over the Amazon Basin from the AERONET network have been reviewed by Schafer et al. (2008). In Table 1 I give the averages from the cleanest 3 months (April-Juni in the northern part of the Amazon Basin, February-April in the southern part) to represent clean conditions, and the average of August and September in the southern part of the Amazon forest to represent the smoky period. The average values for the clean period fall near 0.09 , while in the smoky period they range around 0.90 , and thus show about the same factor-of-ten increase from clean to smoky conditions as the $\mathrm{CCN}$ concentrations. During some of the cleanest episodes, values as low as 0.05 were observed. The Ångstrom exponent, å, during the clean periods is relatively low $(0.7-1.1)$, indicating that coarse particles (primary biogenic material, but also some dust from long-range transport) contribute significantly to AOT. The results from the Amazon forest are nearly identical to values observed at tropical and subtropical marine sites (cf. Table 2 and Fig. 1), such as Lanai (Hawaii) and San Nicolas Island (California), where $\mathrm{AOT}_{500}$ values of $0.08 \pm 0.03$ have been observed, with å in the range of 0.6 to 1.3 (Holben et al., 2001).

During the smoky period, å increases to $1.7 \pm 0.1$ as a result of the increased importance of the fine mode aerosol. The single scattering albedo of the smoke aerosol over the Amazon forest is $0.92 \pm 0.01$, with excellent agreement between sunphotometer and in-situ measurements (Schmid et al., 2006; Schafer et al., 2008).

\section{Remote temperate continental regions}

Because the large expanses of temperate ecosystems fall into the same latitude belt as the regions with the highest density of anthropogenic emissions, it is very difficult to estimate pristine CCN concentrations and AOT over them. There is a surprisingly small number of data sets from remote regions in the temperate zone, and none where CCN and AOT measurements are truly collocated. Furthermore, the direct CCN measurements must either be taken from older studies, with sometimes uncertain measurement accuracy, or be deduced from size distributions. In spite of these problems, a surprisingly consistent picture emerges. In western and northern North America, remote sites tend to have $\mathrm{CCN}_{0.4}$ concentrations ranging from about 90 in winter to ca. 280 in summer. Average $\mathrm{AOT}_{500}$ values typically fall in the $0.06-$ 0.12 range. The northern European $\mathrm{CCN}$ concentrations tend to be somewhat higher, which is not altogether surprising considering the likely impact of residual air pollution in this region (Putaud et al., 2004; Van Dingenen et al., 2004). Unfortunately, no AOT data are available from this region.

An area of great potential interest for aerosol studies are the remote regions of Siberia, where the first measurements with modern aerosol size spectrometers have recently been reported (Heintzenberg et al., 2008). A preliminary analysis of a one-year data set from the ZOTTO tall tower site near $60^{\circ} \mathrm{N}, 90^{\circ} \mathrm{E}$ shows an average $\mathrm{CCN}_{0.4}$ concentration (calculated from $\mathrm{CN}>60 \mathrm{~nm}$ ) of about $190 \mathrm{~cm}^{-3}$ during clean periods (defined as those times when the aerosol absorption coefficient is $<2 \mathrm{Mm}^{-1}$, corresponding to an equivalent black carbon concentration of about $<0.2 \mu \mathrm{g} \mathrm{m}^{-3}$ ). The data show that $\mathrm{CN}$ and light absorption by soot particles are still correlated even at low concentrations, indicating the presence of a residual pollution component. It is difficult to find corresponding AOT data, as the two nearest AERONET sites are in Krasnoyarsk and Tomsk, two highly industrialized cities with strong local pollution sources. The median and quartile ranges for $\mathrm{AOT}_{500}$ at these sites are $0.163(0.122,0.219)$ and $0.138(0.094,0.209)$, respectively. A better site to represent remote values in boreal Siberia may be Yakutsk, which has 


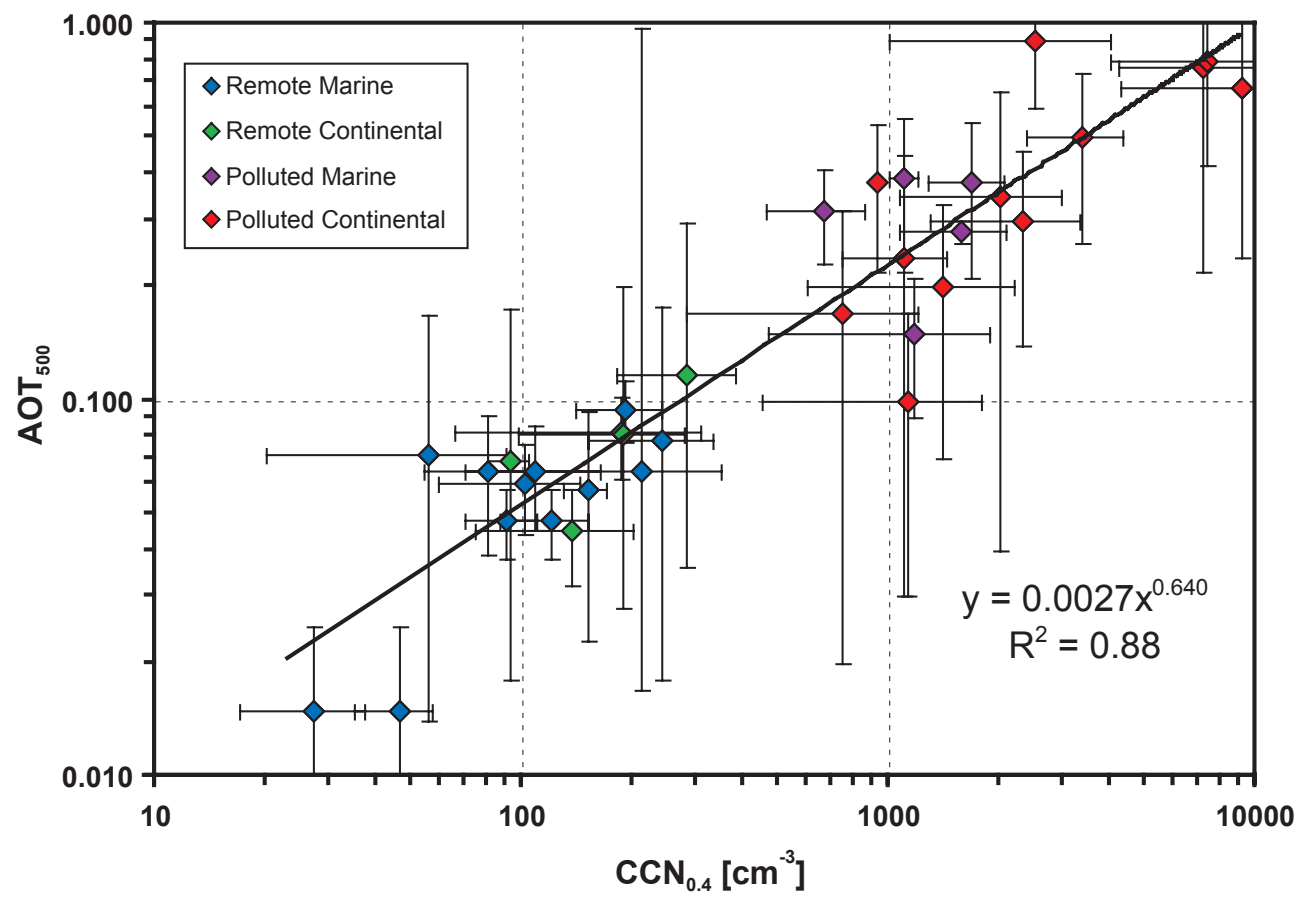

Fig. 1. Relationship between $\mathrm{AOT}_{500}$ and $\mathrm{CCN}_{0.4}$ from investigations where these variables have been measured simultaneously, or where data from nearby sites at comparable times were available. The error bars reflect the variability of measurements within each study (standard deviations or quartiles).

a much smaller population and little industry. The median $\mathrm{AOT}_{500}$ there is $0.081(0.053-0.120)$, comparable to many other remote continental sites, but still considerably higher than at Fort McMurray in northern Canada: 0.057(0.034, 0.089). The only report from the extratropical continental Southern Hemisphere is from a flight campaign in South Africa, where $\mathrm{CCN}_{0.4}$ values of $137 \pm 63$ were measured over the Highveld region on a clean day (Ross et al., 2003). On the same day, the AERONET site at Bethlehem, in the center of the Highveld, measured an $\mathrm{AOT}_{500}$ of $0.045 \pm 0.013$.

In summary, the mean values of $\mathrm{CCN}_{0.4}$ and $\mathrm{AOT}_{500}$ over extratropical remote sites are not distinctly different from those measured over Amazonia, even considering that some influence from long-range transport of pollution aerosol is unavoidable in these measurements. For example, more than half of the sulfate aerosol over remote British Columbia is from East Asian sources (van Donkelaar et al., 2008). This implies that pre-anthropogenic $\mathrm{CCN}_{0.4}$ concentrations over most continental regions were below, maybe even well below, $200 \mathrm{~cm}^{-3}$.

\section{Remote marine regions}

Remote marine regions, especially in the Southern Hemisphere, are usually considered the least polluted and most pristine parts of the atmosphere, but it must be remembered that anthropogenic pollution reaches even the remotest sites. This is, for example, readily seen at the Cape Grim background station, where aerosols from biomass burning in Southern Africa are readily detected during the fire season (Heintzenberg and Bigg, 1990). Nevertheless, remote marine sites show the lowest number concentrations of aerosol particles and $\mathrm{CCN}$ worldwide. In the winter season, $\mathrm{CCN}$ concentrations in some areas drop down to a few tens per $\mathrm{cm}^{3}$, as shown in Table 2 by data from the Southern Ocean (Cape Grim) and the Northeast Pacific (off Washington State), while in other areas, e.g., at the Northeast Atlantic coast (Mace Head) this seasonal cycle is less pronounced. Over biologically productive ocean regions, such as the tropical oceans and the temperate regions in summer, $\mathrm{CCN}_{0.4}$ concentrations are typically near or above $100 \mathrm{~cm}^{-3}$. This seasonal behavior is related to the biogenic production of marine aerosols, probably both via the emission of DMS and its oxidation to sulfate, and the release of primary biogenic particles (Charlson et al., 1987; Andreae and Rosenfeld, 2008). The reduced seasonality at some sites may be related to the effect of low levels of anthropogenic pollution, which can make a significant contribution to the very low aerosol concentrations present at remote oceanic sites in winter (Andreae et al., 1999, 2003; Reade et al., 2006).

Overall, one finds that $\mathrm{CCN}$ concentrations over the present-day remote oceans are on average about one-half of those over the present-day remote continents (Table 2), but with a very broad overlap (Fig. 1). In view of the fact that the 
sources of anthropogenic emissions are all located on land (with the exception of ship-stack emissions), it must be assumed that this ocean-land difference in $\mathrm{CCN}$ concentrations was substantially lower in pre-human times.

Remote ocean areas also show very low AOT values, in spite of the relatively high fraction of scattering associated with the seasalt aerosol (Quinn and Coffman, 1999), and the high AOTs reported in one study that included very high wind speeds (Mulcahy et al., 2008). The datasets compiled in Table 2 yield an average $\mathrm{AOT}_{500}$ of $0.055 \pm 0.023$, in good agreement with the "baseline" marine $\mathrm{AOT}_{550}$ of $0.06 \pm 0.01$ given by Kaufman et al. (2005). Interestingly, the seasonality of AOT at Cape Grim is opposite to that of seasalt aerosol, which has its highest concentrations in the winter (Andreae et al., 1999). This argues against a dominant role of seasalt in controlling AOT, and suggests that the fine aerosol fraction may dominate both CCN concentration and AOT. The same conclusion was reached by Vallina et al. (2006) based on a statistical analysis of the CCN concentrations over the Southern Ocean and their controlling variables.

\section{Polluted continental and marine regions}

CCN concentrations and AOT values in polluted regions show a continuous increase from near-pristine values to extremely high levels in urban or biomass-burning regions (Table 2). In general, polluted marine regions tend to have lower values (range of study averages: $\mathrm{CCN}_{0.4} 600-1700 \mathrm{~cm}^{-3}$, $\mathrm{AOT}_{500}$ 0.15-0.39) than continental polluted areas $\left(\mathrm{CCN}_{0.4}\right.$ $370-9100 \mathrm{~cm}^{-3}, \mathrm{AOT}_{500} 0.10-0.90$ ) because they are usually more distant from sources. This can also be seen at some coastal sites, such as Amnyeon and Gosan (Korea), which experience both direct continental flow and inflow of polluted airmasses that have spent up to several days over the ocean. Thus, the $\mathrm{CCN}_{0.4}$ concentrations in these airmasses, which in terms of airmass trajectories would be classified as marine, are in the range conventionally thought of as "continental", again putting in question the validity of this classification (Roberts et al., 2001). On the other hand, some of the continental sites in North America and Europe (e.g., the New Hampshire, North Carolina, and Hohenpeissenberg sites) show relatively low $\mathrm{CCN}$ and AOT values, most likely as a result of the reductions in pollutant emissions over the last two decades. The highest values in Table 2 come from peri-urban regions in China, i.e., from locations just outside the urban areas of Guangzhou and Beijing. I have not included any urban measurements, because it is difficult to obtain representative measurements in such a highly variable environment, and because this analysis is mainly directed toward the regional to global scale.

\section{Relationship between $\mathrm{CCN}_{0.4}$ and $\mathrm{AOT}_{500}$}

The scatterplot between $\mathrm{CCN}_{0.4}$ and $\mathrm{AOT}_{500}$ (Fig. 1) shows a surprisingly tight relationship, which can be fitted with a power law $\mathrm{AOT}_{500}=0.0027 \cdot\left[\mathrm{CCN}_{0.4}\right]^{0.640}$ with a very high degree of correlation $\left(r^{2}=0.88\right)$. While the deviations of individual studies from this trend are sometimes large (up to a factor of three), and obviously deviations for single measurements at one place and time must be expected to be even greater, this does provide a basis for a parameterization of $\mathrm{CCN}$ concentrations in large-scale regional and global climate models. Note that in almost all cases the regression line goes through the error bars of the data. Figure 1 also highlights the broad overlap between remote marine and continental values.

Some further developments to this approach suggest themselves. Given that the $\mathrm{CCN}$ concentration is more closely tied to the finer fraction of the aerosol, AOT measurements at lower wavelengths might provide better correlations than the commonly used $\mathrm{AOT}_{500}$, which was employed in this study. Alternatively, instead of $\mathrm{AOT}_{500}$, one might examine correlations between $\mathrm{CCN}$ and the aerosol index AI, defined as the product of AOT and the Ångstrom exponent, thus providing another way of weighting the AOT measurement toward the fine mode. Finally, instead of the use of ground-based AOT measurements, one could examine the use of products based on satellite remote sensing, such as the fine mode AOT product from MODIS (Remer et al., 2005), especially once the remaining calibration issues in the MODIS products have been resolved (Remer et al., 2008). These investigations go beyond the scope of the present study, however, which has been designed as a first examination of large-scale relationships between potential proxies for the radiative and cloud microphysical forcings of anthropogenic aerosols.

Figure 2 shows the relationship between $\mathrm{CCN}_{0.4}$ and $\mathrm{CN}$ concentrations. Again, there is a surprisingly good correlation, especially in view of the very different regimes from which the data are taken and the many different instruments by which they have been collected. The data in Table 2 suggest a fairly constant $\mathrm{CCN}_{0.4} / \mathrm{CN}$ ratio of $0.36 \pm 0.14$ (excluding the two very low values from Laramie, Wyoming), which has been plotted as a line in Fig. 2. This relationship reflects the relatively narrow range of hygroscopicity parameters and the convergent character of aerosol size distributions typical of many non-urban regions (Andreae and Rosenfeld, 2008).

\section{Summary and conclusions}

Analysis of published and unpublished data on $\mathrm{AOT}_{500}$ and $\mathrm{CCN}$ concentration shows that measurements from remote oceanic and continental regions fall into relatively narrow ranges. Remote marine $\mathrm{CCN}_{0.4}$ concentrations are typically near or slightly above $100 \mathrm{~cm}^{-3}$ in biologically productive regions and seasons, and of the order of a few tens per $\mathrm{cm}^{3}$ 


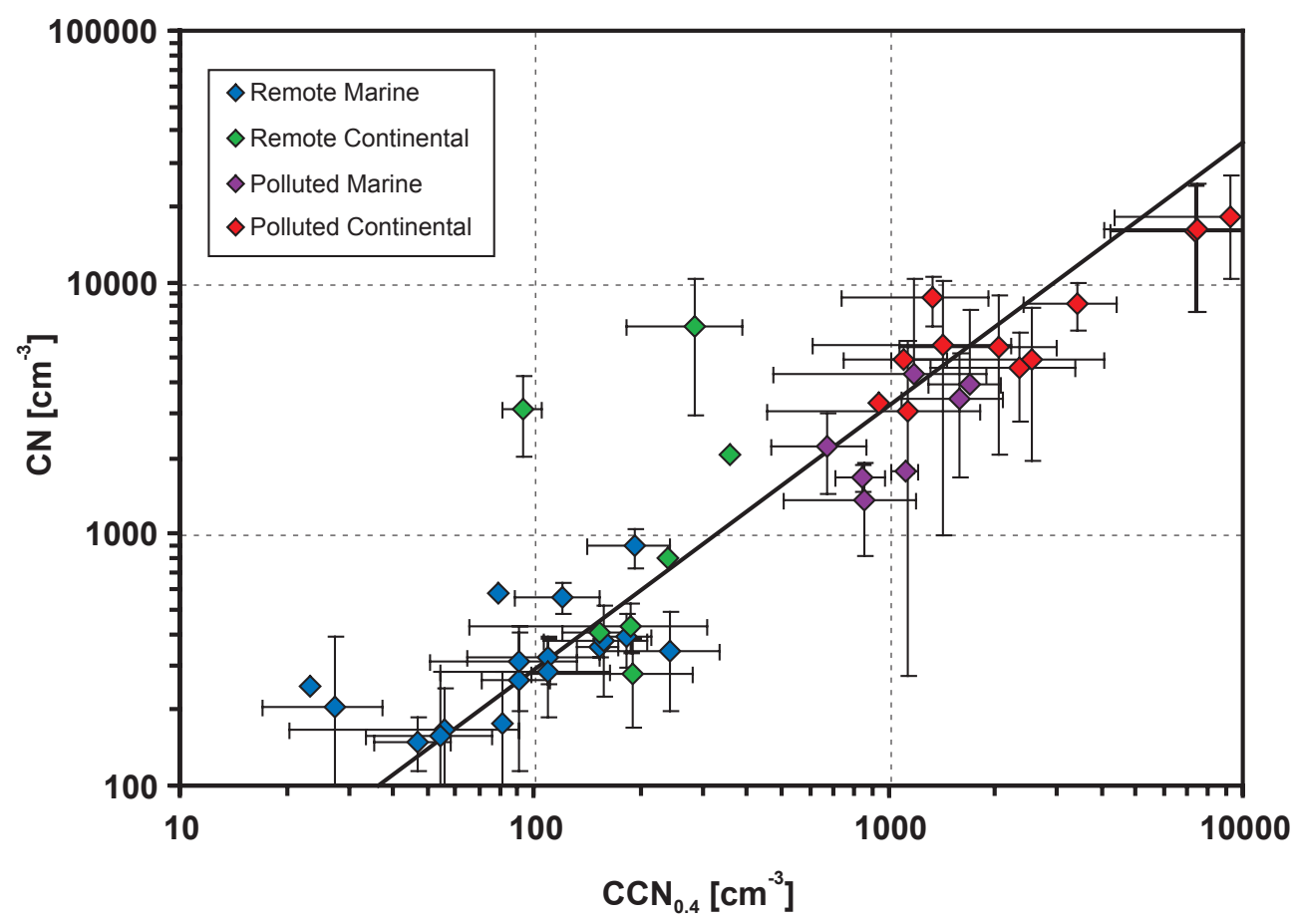

Fig. 2. Scatterplot of $\mathrm{CN}$ vs. $\mathrm{CCN}_{0.4}$ based on the data from Table 2. The line represents the mean $\mathrm{CCN}_{0.4} / \mathrm{CN}$ ratio of 0.36 .

in winter. The average $\mathrm{AOT}_{500}$ over the remote oceans is $0.057 \pm 0.023$, again with lower values in winter. Remote continental areas have, on average, almost twice as many $\mathrm{CCN}$, and a mean $\mathrm{AOT}_{500}$ of $0.075 \pm 0.025$ (Table 2). Some, or maybe even most of this difference, must be related to the closer proximity that even remote continental sites have to pollution sources, underscoring that the difference between marine and continental levels must have been smaller before the advent of anthropogenic pollution. Support for this statement comes from observations in remote regions that show the presence of residual pollution aerosols even under quite clean conditions, from analyses of the spatial patterns of AOT over continents and oceans, and from global aerosol modeling studies (Andreae, 2007a, b; Andreae and Rosenfeld, 2008). CCN concentrations over polluted regions are on average about one order of magnitude greater than over their remote counterparts, while the $\mathrm{AOT}_{500}$ values over the polluted regions are about 5 times those over their remote equivalents (Table 2).

$\mathrm{CCN}_{0.4}$ concentrations and $\mathrm{AOT}_{500}$ values show a surprising degree of correlation, which can be expressed as a power law (note that regions dominated by desert dust have been excluded from this analysis). Given the difficulty of making direct $\mathrm{CCN}$ measurements, this relationship should be of great practical value in large-scale studies on the influence of the various direct and indirect aerosol effects on climate, as it provides an easily measured proxy for $\mathrm{CCN}$ concentrations. It is obvious, that the use of such a relationship requires a number of caveats, in particular in relation to the statistical nature of the relationship, which includes the considerable variability that must be expected at any given time and place due to the effects of the vertical structure of the aerosol distribution, the influence of relative humidity, the presence of large amounts of mineral dust, and other factors. Various refinements to this analysis can be suggested, including the use of different AOT measurement wavelengths and spaceborne remote sensing.

As noted in the introduction, this study was motivated by the desire to explore the range of values typical of various aerosol regimes in the present-day atmosphere, based on a compilation of observations, with the intention of using the results in modeling studies. The analysis presented here shows that the selected proxies for the microphysical and radiative effects of clouds on aerosols, $\mathrm{CCN}_{0.4}$ and $\mathrm{AOT}_{500}$, do not vary fully independently of one another. This implies that the radiative and microphysical effects of aerosols on clouds, and therefore on climate and precipitation, are correlated and should not be treated independently of one another, at least not on larger scales. Further discussion of this conceptual approach and initial results can be found in the recent paper by Rosenfeld et al. (2008).

Acknowledgements. I thank D. Rosenfeld for stimulating discussions that led to this analysis, as well as the Integrated Land Ecosystem-Atmosphere Process Study (iLEAPS) and the International Space Studies Institute (ISSI) for providing a forum for discussions that stimulated this paper. I am grateful to J. Gras, S. Gunthe, G. Frank, D. Rose, U. Dusek, H. Yang, U. Pöschl, N. Jürgens, J. Schneider, P. Artaxo, S. Martin, M. Kulmala, 
W. Birmili, A. Wiedensohler, and J. Heintzenberg for letting me use their unpublished $\mathrm{CCN}$ and aerosol data. I thank the following AERONET investigators and their staff for establishing and maintaining the sites used in this investigation and for making their data available: B. Holben, W.-M. Hao, B. McArthur, M. Panchenko, P. Goloub, R. Frouin, O. Mayol-Bracero, E. Cuevas, S. Jones, P.-H. Lin, J. E. Nichol, A. Royer, N. O’Neill, S.-C. Yoon.

Edited by: W. Conant

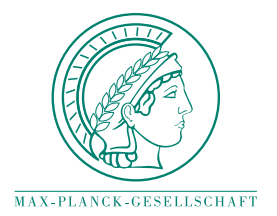

This Open Access Publication is financed by the Max Planck Society.

\section{References}

Allan, J. D., Baumgardner, D., Raga, G. B., Mayol-Bracero, O. L., Morales-García, F., García-García, F., Montero-Martínez, G., Borrmann, S., Schneider, J., Mertes, S., Walter, S., Gysel, M., Dusek, U., Frank, G. P., and Krämer, M.: Clouds and aerosols in Puerto Rico - a new evaluation, Atmos. Chem. Phys., 8, 12931309, 2008, http://www.atmos-chem-phys.net/8/1293/2008/.

Andreae, M. O.: Aerosols before pollution: Science, 315, 50-51, 2007a.

Andreae, M. O.: Atmospheric aerosols versus greenhouse gases in the 21st century, Phil. Trans. R. Soc. A, 365, 1915-1923, $2007 \mathrm{~b}$.

Andreae, M. O., Andreae, T. W., Meyerdierks, D., and Thiel, C.: Marine sulfur cycling and the atmospheric aerosol over the springtime North Atlantic, Chemosphere, 52, 1321-1343, 2003.

Andreae, M. O., Artaxo, P., Brandão, C., et al.: Biogeochemical cycling of carbon, water, energy, trace gases and aerosols in Amazonia: The LBA-EUSTACH experiments, J. Geophys. Res., 107, 8066, doi:10.1029/2001JD000524, 2002.

Andreae, M. O., Berresheim, H., Bingemer, H., Jacob, D. J., Lewis, B. L., Li, S.-M., and Talbot, R. W.: The atmospheric sulfur cycle over the Amazon Basin, 2. Wet Season, J. Geophys. Res., 95, 16813-16824, 1990.

Andreae, M. O., Elbert, W., Cai, Y., Andreae, T. W., and Gras, J.: Non-seasalt sulfate, methanesulfonate, and nitrate aerosol concentrations and size distributions at Cape Grim, Tasmania, J. Geophys. Res., 104, 21695-21706, 1999.

Andreae, M. O. and Rosenfeld, D.: Aerosol-cloud-precipitation interactions, Part 1, The nature and sources of cloud-active aerosols: Earth Science Reviews, 89, 13-41, 2008.

Andreae, M. O., Rosenfeld, D., Artaxo, P., Costa, A. A., Frank, G. P., Longo, K. M., and Silva-Dias, M. A. F.: Smoking rain clouds over the Amazon, Science, 303, 1337-1342, 2004.

Artaxo, P., Martins, J. V., Yamasoe, M. A., Procópio, A. S., Pauliquevis, T. M., Andreae, M. O., Guyon, P., Gatti, L. V., and Leal, A. M. C.: Physical and chemical properties of aerosols in the wet and dry season in Rondonia, Amazonia, J. Geophys. Res., 107, 8081, doi:10.1029/2001JD000666, 2002.

Birmili, W., Berresheim, H., Plass-Dülmer, C., Elste, T., Gilge, S., Wiedensohler, A., and Uhrner, U.: The Hohenpeissenberg aerosol formation experiment (HAFEX): a long-term study including size-resolved aerosol, $\mathrm{H}_{2} \mathrm{SO}_{4}, \mathrm{OH}$, and monoterpenes measurements, Atmos. Chem. Phys., 3, 361-376, 2003, http://www.atmos-chem-phys.net/3/361/2003/.

Charlson, R. J., Lovelock, J. E., Andreae, M. O., and Warren, S. G.: Oceanic phytoplankton, atmospheric sulphur, cloud albedo, and climate, Nature, 326, 655-661, 1987.

Delene, D. J. and Deshler, T.: Vertical profiles of cloud condensation nuclei above Wyoming, J. Geophys. Res., 106, 1257912588, 2001.

Dubovik, O., Smirnov, A., Holben, B. N., King, M. D., Kaufman, Y. J., Eck, T. F., and Slutsker, I.: Accuracy assessments of aerosol optical properties retrieved from Aerosol Robotic Network (AERONET) Sun and sky radiance measurements, J. Geophys. Res., 105, 9791-9806, 2000.

Formenti, P., Andreae, M. O., Lange, L., Roberts, G., Cafmeyer, J., Rajta, I., Maenhaut, W., Holben, B. N., Artaxo, P., and Lelieveld, J.: Saharan dust in Brazil and Suriname during the Large-Scale Biosphere-Atmosphere Experiment in Amazonia (LBA)- Cooperative LBA Regional Experiment (CLAIRE) in March 1998, J. Geophys. Res., 106, 14919-14934, 2001.

Frank, G. P., Dusek, U., and Andreae, M. O.: Technical Note: Characterization of a static thermal-gradient CCN counter, Atmos. Chem. Phys., 7, 3071-3080, 2007, http://www.atmos-chem-phys.net/7/3071/2007/.

Gasso, S. and Hegg, D. A.: On the retrieval of columnar aerosol mass and CCN concentration by MODIS, J. Geophys. Res., 108, 4010, doi:10.1029/2002JD002382, 2003.

Ghan, S. J., Rissman, T. A., Elleman, R., Ferrare, R. A., Turner, D., Flynn, C., Wang, J., Ogren, J., Hudson, J., Jonsson, H. H., VanReken, T., Flagan, R. C., and Seinfeld, J. H.: Use of in situ cloud condensation nuclei, extinction, and aerosol size distribution measurements to test a method for retrieving cloud condensation nuclei profiles from surface measurements, J. Geophys Res., 111, D05S10, doi:10.1029/2004JD005752, 2006.

Gras, J. L.: Cloud condensation nuclei over the Southern Ocean, Geophys. Res. Lett., 17, 1565-1567, 1990.

Guyon, P., Graham, B., Beck, J., Boucher, O., Gerasopoulos, E., Mayol-Bracero, O. L., Roberts, G. C., Artaxo, P., and Andreae, M. O.: Physical properties and concentration of aerosol particles over the Amazon tropical forest during background and biomass burning conditions, Atmos. Chem. Phys., 3, 951-967, 2003, http://www.atmos-chem-phys.net/3/951/2003/.

Hegg, D. A., Radke, L. F., and Hobbs, P. V.: Measurements of Aitken nuclei and cloud condensation nuclei in the marine atmosphere and their relation to the DMS-cloud-climate hypothesis, J. Geophys. Res., 96, 18727-18733, 1991.

Heintzenberg, J. and Bigg, E. K.: Tropospheric transport of trace substances in the southern hemisphere, Tellus, 42B, 355-363, 1990.

Heintzenberg, J., Birmili, W., Theiss, D., and Kisilyakhov, Y.: The atmospheric aerosol over Siberia, as seen from the $300 \mathrm{~m}$ ZOTTO tower, Tellus, 60B, 276-285, doi:10.1111/j.16000889.2007.0035.x, 2008.

Holben, B. N., Tanre, D., Smirnov, A., et al.: An emerging ground-based aerosol climatology: Aerosol optical depth from AERONET, J. Geophys. Res., 106, 12067-12097, 2001.

Hoppel, W. A., Dinger, J. E., and Ruskin, R. E.: Vertical profiles of $\mathrm{CCN}$ at various geographical locations, J. Atmos. Sci., 30, 14101420, 1973.

Hudson, J. G.: Cloud condensation nuclei near marine cumulus, J. 
Geophys. Res., 98, 2693-2702, 1993.

Hudson, J. G. and Frisbie, P. R.: Cloud condensation nuclei near marine stratus, J. Geophys. Res., 96, 20795-20808, 1991a.

Hudson, J. G. and Frisbie, P. R.: Surface cloud condensation nuclei and condensation nuclei measurements at Reno, Nevada, Atmos. Environ., 25A, 2285-2299, 1991b.

Hudson, J. G. and Xie, Y. H.: Vertical distributions of cloud condensation nuclei spectra over the summertime northeast Pacific and Atlantic Oceans, J. Geophys. Res., 104, 30219-30229, 1999.

Hudson, J. G., Xie, Y. H., and Yum, S. S.: Vertical distributions of cloud condensation nuclei spectra over the summertime Southern Ocean, J. Geophys. Res., 103, 16609-16624, 1998.

Hudson, J. G. and Yum, S. S.: Cloud condensation nuclei spectra and polluted and clean clouds over the Indian Ocean, J. Geophys. Res., 107, 8022, doi:10.1029/2001JD000829, 2002.

IAPSAG, WMO/IUGG International Aerosol Precipitation Science Assessment Group (IAPSAG) Report: Aerosol Pollution Impact on Precipitation: A Scientific Review, World Meteorological Organization, 2007.

IPCC, Climate Change 2007: The Physical Science Basis, Contribution of Working Group I to the Fourth Assessment Report of the Intergovernmental Panel on Climate Change, edited by: Solomon, S., Qin, D., Manning, M., Chen, Z., Marquis, M., Averyt, K., Tignor, M., and Miller, H. L.: 996 pp., Cambridge University Press, Cambridge and New York, 2007.

Jennings, S. G., Geever, M., and O'Connor, T. C.: Coastal CCN measurements at Mace Head with enhanced concentrations in strong winds, Atmos. Res., 46, 243-252, 1998.

Johnson, D. W., Osborne, S. R., Wood, R., Suhre, K., Quinn, P. K., Bates, T., Andreae, M. O., Noone, K. J., Glantz, P., Bandy, B., Rudolph, J., and O'Dowd, C.: Observations of the evolution of the aerosol, cloud and boundary-layer characteristics during the 1st ACE-2 Lagrangian experiment, Tellus, 52B, 348-374, 2000.

Kahn, R. A., Garay, M. J., Nelson, D. L., Yau, K. K., Bull, M. A., Gaitley, B. J., Martonchik, J. V., and Levy, R. C.: Satellite-derived aerosol optical depth over dark water from MISR and MODIS: Comparisons with AERONET and implications for climatological studies, J. Geophys. Res., 112, D18205, doi:10.1029/2006JD008175, 2007.

Kapustin, V. N., Clarke, A. D., Shinozuka, Y., Howell, S., Brekhovskikh, V., Nakajima, T., and Higurashi, A.: On the determination of a cloud condensation nuclei from satellite: Challenges and possibilities, J. Geophys. Res., 111, D04202, doi:10.1029/2004JD005527, 2006.

Kaufman, Y. J., Boucher, O., Tanre, D., Chin, M., Remer, L. A., and Takemura, T.: Aerosol anthropogenic component estimated from satellite data, Geophys. Res. Lett., 32, L17804, doi:10.1029/2005GL023125, 2005.

Kaufman, Y. J., Tanré, D., and Boucher, O.: A satellite view of aerosols in the climate system, Nature, 419, 215-223, 2002.

Kim, S. W., Yoon, S. C., Dutton, E. G., Kim, J., Wehrli, C., and Holben, B. N.: Global surface-based sun photometer network for long-term observations of column aerosol optical properties: Intercomparison of aerosol optical depth, Aerosol Sci. Tech., 42, $1-9,2008$

Kokhanovsky, A. A., Breon, F. M., Cacciari, A., Carboni, E., Diner, D., Di Nicolantonio, W., Grainger, R. G., Grey, W. M. F., Holler, R., Lee, K. H., Li, Z., North, P. R. J., Sayer, A. M., Thomas, G. E., and von Hoyningen-Huene, W.: Aerosol remote sensing over land: A comparison of satellite retrievals using different algorithms and instruments, Atmos. Res., 85, 372-394, 2007.

Komppula, M., Lihavainen, H., Kerminen, V. M., Kulmala, M., and Viisanen, Y.: Measurements of cloud droplet activation of aerosol particles at a clean subarctic background site, J. Geophys. Res., 110, D06204, doi:10.1029/2004JD005200, 2005.

Li, Z. Q., Niu, F., Lee, K. H., Xin, J. Y., Hao, W. M., Nordgren, B., Wang, Y. S., and Wang, P. C.: Validation and understanding of moderate resolution imaging spectroradiometer aerosol products (C5) using ground-based measurements from the handheld Sun photometer network in China, J. Geophys. Res., 112, D22S07, doi:10.1029/2007JD008479, 2007.

Lihavainen, H., Kerminen, V. M., Komppula, M., Hatakka, J., Aaltonen, V., Kulmala, M., and Viisanen, Y.: Production of "potential" cloud condensation nuclei associated with atmospheric new-particle formation in northern Finland, J. Geophys. Res., 108, 4782, doi:10.1029/2003JD003887, 2003.

Liu, P. S. K., Leaitch, W. R., Banic, C. M., and Li, S.-M.: Aerosol observations at Chebogue Point during the 1993 North Atlantic Regional Experiment: Relationships among cloud condensation nuclei, size distribution, and chemistry, J. Geophys. Res., 101, 28971-28990, 1996.

Lohmann, U. and Feichter, J.: Global indirect aerosol effects: a review, Atmos. Chem. Phys., 5, 715-737, 2005, http://www.atmos-chem-phys.net/5/715/2005/.

McFiggans, G., Artaxo, P., Baltensperger, U., Coe, H., Facchini, M. C., Feingold, G., Fuzzi, S., Gysel, M., Laaksonen, A., Lohmann, U., Mentel, T. F., Murphy, D. M., O’Dowd, C. D., Snider, J. R., and Weingartner, E.: The effect of physical and chemical aerosol properties on warm cloud droplet activation, Atmos. Chem. Phys., 6, 2593-2649, 2006, http://www.atmos-chem-phys.net/6/2593/2006/.

Medina, J., Nenes, A., Sotiropoulou, R. E. P., Cottrell, L. D., Ziemba, L. D., Beckman, P. J., and Griffin, R. J.: Cloud condensation nuclei closure during the International Consortium for Atmospheric Research on Transport and Transformation 2004 campaign: Effects of size-resolved composition, J. Geophys. Res., 112, D10S31, doi:10.1029/2006JD007588, 2007.

Mulcahy, J. P., O’Dowd, C., Jennings, S. G., and Ceburnis, D.: Significant enhancement of aerosol optical depth in marine air under high wind conditions, Geophys. Res. Lett., 35, L16810, doi:10.1029/2008GL034303, 2008.

Osborne, S. R., Johnson, D. W., Wood, R., Bandy, B. J., Andreae, M. O., O’Dowd, C. D., Glantz, P., Noone, K., Gerbig, C., Rudolph, J., Bates, T. S., and Quinn, P.: Evolution of the aerosol, cloud, and boundary layer dynamic and thermodynamic characteristics during the second Lagrangian experiment of ACE-2, Tellus, 52B, 375-400, 2000.

Penner, J. E., Andreae, M. O., Annegarn, H., Barrie, L., Feichter, J., Hegg, D., Jayaraman, A., Leaitch, R., Murphy, D., Nganga, J., and Pitari, G.: Aerosols, their Direct and Indirect Effects, in: Climate Change 2001: The Scientific Basis, Contribution of Working Group I to the Third Assessment Report of the Intergovernmental Panel on Climate Change, edited by: J. T. Houghton, Ding, Y., Griggs, D. J., Noguer, M., van der Linden, P. J., Dai, X., Maskell, K., and Johnson, C. A., Cambridge University Press, Cambridge, UK, and New York, NY, USA, 289-348, 2001.

Petters, M. D. and Kreidenweis, S. M.: A single parameter representation of hygroscopic growth and cloud condensation nucleus 
activity, Atmos. Chem. Phys., 7, 1961-1971, 2007, http://www.atmos-chem-phys.net/7/1961/2007/.

Prospero, J. M., Glaccum, R. A., and Nees, R. T.: Atmospheric transport of soil dust from Africa to South America, Nature, 289, 570-572, 1981.

Putaud, J. P., Raes, F., Van Dingenen, R., et al.: European aerosol phenomenology - 2: chemical characteristics of particulate matter at kerbside, urban, rural and background sites in Europe, Atmos. Environ., 38, 2579-2595, 2004.

Quinn, P. K. and Coffman, D. J.: Comment on "Contribution of different aerosol species to the global aerosol extinction optical thickness: Estimates from model results" by Tegen et al., J. Geophys. Res., 104, 4241-4248, 1999.

Reade, L., Jennings, S. G., and McSweeney, G.: Cloud condensation nuclei measurements at Mace Head, Ireland, over the period 1994-2002, Atmos. Res., 82, 610-621, doi:10.1016/j.atmosres.2006.02.017, 2006.

Remer, L. A., Kaufman, Y. J., Tanre, D., Mattoo, S., Chu, D. A., Martins, J. V., Li, R. R., Ichoku, C., Levy, R. C., Kleidman, R. G., Eck, T. F., Vermote, E., and Holben, B. N.: The MODIS aerosol algorithm, products, and validation, J. Atmos. Sci., 62, 947-973, 2005.

Remer, L. A., Kleidman, R. G., Levy, R. C., Kaufman, Y. J., Tanre, D., Mattoo, S., Martins, J. V., Ichoku, C., Koren, I., Yu, H. B., and Holben, B. N.: Global aerosol climatology from the MODIS satellite sensors, J. Geophys. Res., 113, D14S07, doi:10.1029/2007JD009661, 2008.

Rissler, J., Swietlicki, E., Zhou, J., Roberts, G., Andreae, M. O., Gatti, L. V., and Artaxo, P.: Physical properties of the submicrometer aerosol over the Amazon rain forest during the wetto-dry season transition - comparison of modeled and measured CCN concentrations, Atmos. Chem. Phys., 4, 2119-2143, 2004, http://www.atmos-chem-phys.net/4/2119/2004/.

Roberts, G. C., Andreae, M. O., Zhou, J., and Artaxo, P.: Cloud condensation nuclei in the Amazon Basin: "Marine" conditions over a continent?, Geophys. Res. Lett., 28, 2807-2810, 2001.

Rose, D., Gunthe, S. S., Mikhailov, E., Frank, G. P., Dusek, U., Andreae, M. O., and Pöschl, U.: Calibration and measurement uncertainties of a continuous-flow cloud condensation nuclei counter (DMT-CCNC): CCN activation of ammonium sulfate and sodium chloride aerosol particles in theory and experiment, Atmos. Chem. Phys., 8, 1153-1179, 2008, http://www.atmos-chem-phys.net/8/1153/2008/.

Rosenfeld, D.: Aerosol-cloud interactions control of earth radiation and latent heat release budgets, Space Sci. Rev., 125, 149-157, doi:10.1007/s11214-006-9053-6, 2006a.

Rosenfeld, D.: Aerosols, Clouds, and Climate, Science, 312, 1323 1324, 2006b.

Rosenfeld, D., Lohmann, U., Raga, G. B., O’Dowd, C. D., Kulmala, M., Fuzzi, S., Reissell, A., and Andreae, M. O.: Flood or drought: How do aerosols affect precipitation?, Science, 321, 1309-1313, 2008.

Ross, K. E., Piketh, S. J., Bruintjes, R. T., Burger, R. P., Swap, R. J., and Annegarn, H. J.: Spatial and seasonal variations in CCN distribution and the aerosol-CCN relationship over southern Africa, J. Geophys. Res., 108, 8481, doi:10.1029/2002JD002384, 2003.

Schäfer, B., Georgii, H.-W., and Staubes, R.: Formation and distribution of cloud condensation nuclei in the marine environment, in: Dimethylsulphide: Oceans, Atmosphere, and Climate, edited by: Restelli, G. and Angeletti, G., Kluwer, Dordrecht, 297-302, 1993.

Schafer, J. S., Eck, T. F., Holben, B. N., Artaxo, P., and Duarte, A. F.: Characterization of the optical properties of atmospheric aerosols in Amazônia from long-term AERONET monitoring (1993-1995 and 1999-2006), J. Geophys. Res., 113, D04204, doi:10.1029/2007JD009319, 2008.

Schmid, O., Artaxo, P., Arnott, W. P., Chand, D., Gatti, L. V., Frank, G. P., Hoffer, A., Schnaiter, M., and Andreae, M. O.: Spectral light absorption by ambient aerosols influenced by biomass burning in the Amazon Basin. I: Comparison and field calibration of absorption measurement techniques, Atmos. Chem. Phys., 6, 3443-3462, 2006,

http://www.atmos-chem-phys.net/6/3443/2006/.

Seinfeld, J. H. and Pandis, S. N.: Atmospheric chemistry and physics: From air pollution to climate change, New York, John Wiley, 1326 pp., 1998.

Squires, P. and Twomey, S.: A comparison of cloud nucleus measurements over central North America and the Caribbean Sea, J. Atmos. Sci., 23, 401-404, 1966.

Stroud, C. A., Nenes, A., Jimenez, J. L., DeCarlo, P. F., Huffman, J. A., Bruintjes, R., Nemitz, E., Delia, A. E., Toohey, D. W., Guenther, A. B., and Nandi, S.: Cloud activating properties of aerosol observed during CELTIC, J. Atmos. Sci., 64, 441-459, 2007.

Swap, R., Garstang, M., Greco, S., Talbot, R., and Kållberg, P.: Saharan dust in the Amazon Basin: Tellus, 44B, 133-149, 1992.

Talbot, R. W., Andreae, M. O., Berresheim, H., Artaxo, P., Garstang, M., Harriss, R. C., Beecher, K. M., and Li, S. M. Aerosol chemistry during the wet season in Central Amazonia: The influence of long-range transport, J. Geophys. Res., 95, $16955-16969,1990$.

Tomlinson, J. M., Li, R. J., and Collins, D. R.: Physical and chemical properties of the aerosol within the southeastern $\mathrm{Pa}$ cific marine boundary layer, J. Geophys. Res., 112, D12211, doi:10.1029/2006JD007771, 2007.

Vallina, S. M., Simo, R., and Gasso, S.: What controls CCN seasonality in the Southern Ocean? A statistical analysis based on satellite-derived chlorophyll and CCN and model-estimated $\mathrm{OH}$ radical and rainfall, Global Biogeochem. Cy., 20, GB1014, doi:10.1029/2005GB002597, 2006

Van Dingenen, R., Raes, F., Putaud, J. P., et al.: A European aerosol phenomenology-1: physical characteristics of particulate matter at kerbside, urban, rural and background sites in Europe, Atmos. Environ., 38, 2561-2577, 2004

van Donkelaar, A., Martin, R. V., Leaitch, W. R., Macdonald, A. M., Walker, T. W., Streets, D. G., Zhang, Q., Dunlea, E. J., Jimenez, J. L., Dibb, J. E., Huey, L. G., Weber, R., and Andreae, M. O.: Analysis of aircraft and satellite measurements from the Intercontinental Chemical Transport Experiment (INTEX-B) to quantify long-range transport of East Asian sulfur to Canada, Atmos. Chem. Phys., 8, 2999-3014, 2008, http://www.atmos-chem-phys.net/8/2999/2008/.

Williams, E., Rosenfeld, D., Madden, N., et al.: Contrasting convective regimes over the Amazon: Implications for cloud electrification, J. Geophys. Res., 107, 8082, doi:10.1029/2001JD000380, 2002.

Wilson, S. R. and Forgan, B. W.: Aerosol optical depth at Cape Grim, Tasmania, 1986-1999, J. Geophys. Res., 107, 4068, 
doi:10.1029/2001JD000398, 2002.

Wood, R., Johnson, D., Osborne, S., Andreae, M. O., Bandy, B., Bates, T. S., O'Dowd, C., Glantz, P., Noone, K., Quinn, P. K., Rudolph, J., and Suhre, K.: Boundary layer and aerosol evolution during the 3rd Lagrangian experiment of ACE-2, Tellus, 52B, 401-422, 2000.

Yu, H. B., Dickinson, R. E., Chin, M., Kaufman, Y. J., Holben, B. N., Geogdzhayev, I. V., and Mishchenko, M. I.: Annual cycle of global distributions of aerosol optical depth from integration of MODIS retrievals and GOCART model simulations, J. Geophys. Res., 108, 4128, doi:10.1029/2002JD002717, 2003.

Yum, S. S. and Hudson, J. G.: Vertical distributions of cloud condensation nuclei spectra over the springtime Arctic Ocean, J. Geophys. Res., 106, 15045-15052, 2001.
Yum, S. S. and Hudson, J. G., Wintertime/summertime contrasts of cloud condensation nuclei and cloud microphysics over the Southern Ocean, J. Geophys. Res., 109, D06204, doi:10.1029/2003JD003864, 2004.

Yum, S. S., Hudson, J. G., Song, K. Y., and Choi, B. C.: Springtime cloud condensation nuclei concentrations on the west coast of Korea, Geophys. Res. Lett., 32, L09814, doi:10.1029/2005GL022641, 2005.

Yum, S. S., Roberts, G., Kim, J. H., Song, K. Y., and Kim, D. Y.: Submicron aerosol size distributions and cloud condensation nuclei concentrations measured at Gosan, Korea, during the Atmospheric brown clouds - East Asian Regional Experiment 2005, J. Geophys. Res., 112, D22S32, doi:10.1029/2006JD008212, 2007. 